\title{
Anatomical variations of the obturator artery in a group of Sri Lankan subjects: A cadaveric study
}

\author{
Samarawickrama $\mathrm{MB}^{1}$, Rodrigo $\mathrm{PM}^{1}$, Nanayakkara $\mathrm{PGCL}^{1}$, Chamara $\mathrm{AGD}^{1}$, Tharaka \\ $\mathrm{NGS}^{1}$, Karunadasa THAS ${ }^{1}$. \\ ${ }^{\prime}$ Department of Anatomy, Faculty of Medicine, University of Ruhuna. \\ *smaramb@gmail.com
}

The aberrant obturator artery (AOA) which crosses the superior pubic ramus is at risk of damage in pelvic trauma and surgeries. Therefore, the knowledge of its common variations and incidence are important in clinical practice. The aim of the study was to identify its variations in a group of Sri Lankan subjects. The study was conducted in the Department of Anatomy, Faculty of Medicine, University of Ruhuna using pelvises of the cadavers during educational dissections. The origin, course and variations of the OA were noted. The study included 30 hemipelvises. There were 8 males and 7 females. The normal pattern of the OA originating from the anterior division of internal iliac artery (IIA) was found in 17 $(56.6 \%)$ hemipelvises. Numerical and morphological variations were seen in $13(43.3 \%)$ hemipelvises. Out of all the hemipelvises $7(23.3 \%)$ OAs originated from the inferior epigastric artery (IEA) while $4(13.3 \%)$ OAs originated from the posterior division of IIA. Double OA was found in 2 hemipelvises. AOA with or without anastomoses with IIA branch crossing the superior pubic ramus was observed in $8(26.6 \%)$ hemipelvises. Most of the variations were present in male subjects [7(53.84\%)]. The data of this study shows that AOA is present in nearly $1 / 3$ rd of the hemipelvises. The knowledge of their pattern and relations are of academic interest to anatomists and clinically important to surgeons in preventing accidental damage during surgery. 